\title{
The value of the pre-hospital learning environment as part of the emergency nursing programme
}

\author{
Sonett van Wyk ${ }^{a, *}$, Tanya Heyns ${ }^{b}$, Isabel Coetzee ${ }^{b}$ \\ a Mediclinic, South Africa, PO Box 102125, Moreleta Plaza, Pretoria, 0167, South Africa \\ b University of Pretoria, South Africa Department of Nursing Science, Private Bag X323, Arcadia, Pretoria, 0007, \\ South Africa
}

\section{A R T I C L E I N F O}

Article history:

Received 12 February 2015

Accepted 8 May 2015

Available online 29 September 2015

Keywords:

Emergency nursing programme

Clinical experience

Pre-hospital environment

\begin{abstract}
A B S T R A C T
Background: Professional nurses enrolled in a post-basic emergency nursing programme presented at a tertiary nursing education institution in South Africa are placed in different clinical learning environments to reach the set clinical outcomes and gain appropriate clinical experience. These students are placed in the hospital environment (emergency department and critical care unit) and pre-hospital environment (road ambulance services) to ensure they gain the necessary clinical exposure in order to learn the relevant and necessary skills and knowledge which in turn will help them become safe and independent emergency nurse practitioners. Controversy about the value of placing emergency nursing students in the pre-hospital environment has raised questions from specifically the private healthcare sector. During the pre-hospital placement, emergency nursing students are absent from the hospital environment which is costly and, according to the healthcare services, unnecessary.

Objective: The study explored the views of the emergency nurse students regarding the value of rotating through the pre-hospital learning environment during an emergency nursing programme.

Methods: A qualitative, explorative, descriptive and contextual research design using an Appreciative Inquiry approach was used to collect the data. Through purposive sampling a total of 45 emergency nursing students participated. Data was collected by means of selfreported Appreciative Inquiry interview guides and individual Appreciative Inquiry interviews. The data was analysed using content analysis.

Results: Four major themes were identified: an unpredictable environment, role players in emergency medical services, team work, and competencies.

Conclusion: The research findings support the value and continuation of utilising the prehospital clinical learning environment for placing post-basic emergency nursing students when enrolled in the emergency nursing programme.

Copyright $\odot$ 2015, The Authors. Production and hosting by Elsevier B.V. on behalf of Johannesburg University. This is an open access article under the CC BY-NC-ND license (http://creativecommons.org/licenses/by-nc-nd/4.0/).
\end{abstract}

* Corresponding author. Mediclinic South Africa Training, 132 Celliers Street, Sunnyside, Pretoria, 0132 , South Africa. Tel.: +2782491 4897.

E-mail addresses: sonett.vanwyk@gmail.com (S. van Wyk), tanya.heyns@up.ac.za (T. Heyns), isabel.coetzee@up.ac.za (I. Coetzee). Peer review under responsibility of Johannesburg University. http://dx.doi.org/10.1016/j.hsag.2015.05.001

1025-9848/Copyright @ 2015, The Authors. Production and hosting by Elsevier B.V. on behalf of Johannesburg University. This is an open access article under the CC BY-NC-ND license (http://creativecommons.org/licenses/by-nc-nd/4.0/). 


\section{Introduction and background}

Essential learning opportunities assist in the development of particular competencies within the nursing profession. These opportunities are provided through clinical learning. Clinical learning is therefore regarded as a vital component of nursing programmes. In South Africa, registered professional nurses working in the emergency nursing environment can enrol for the "Medical and Surgical: Emergency Nursing" post-basic training programme presented at tertiary institutions (Brysiewicz \& Bruce, 2008, p. 129). An emergency nurse practitioner can be defined as a "nurse practising in an emergency department who is specifically trained to deal with minor injuries without the need for supervision by a physician" (Medical dictionary for health professions and nursing, 2012).

The clinical experience gained during exposure to various clinical learning environments promotes the development of the necessary knowledge and skills (Chesser-Smyth, 2005, p. 320; Melby, 2000, p. 638), while planned clinical placement may enhance emergency nursing students' ability to integrate theory with practice (Edwards, Smith, Courtney, Finlayson, \& Chapman, 2004, p. 249; Jackson \& Mannix, 2001, p. 276). Working in the clinical environment promotes quality of learning as this setting is vital to support development towards future quality practices as registered emergency nurses (Meyer \& van Niekerk, 2008, p. 65). Moreover, clinical exposure is regarded as vital for adequate basic and post-basic nursing training as students are exposed to potential learning opportunities (Jerlock, Falk, \& Severinsson, 2003, p. 219; ChunHeung \& French, 1997, p. 460).

An emergency nurse programme presented at a higher education institution in South Africa focuses on particular theoretical content and related clinical exposure as prescribed by the South African Nursing Council (SANC) (R212, 1993). Clinical exposure is based on the available learning opportunities within the clinical learning environment. To comply with the curriculum of the programme, emergency nursing students need to rotate through various clinical learning environments in order to gain competencies in the emergency environment. The pre-hospital environment is in fact only one of a number of different clinical learning environments. In terms of the curriculum of the specific nursing education institution in Gauteng, South Africa, the emergency nursing students have to rotate through the pre-hospital environment for a period of eight weeks. Educators view the eight-week rotation through the prehospital environment as an essential clinical learning opportunity because it engenders appropriate clinical exposure.

However, the placement of emergency nursing students (who are enrolled in the emergency nursing programme) within the pre-hospital learning environment seems to be a controversial issue. During a preceptors meeting, the preceptors shared with the researcher their concern that the placement of emergency nursing students in the prehospital environment for eight weeks is regarded by the healthcare services as financially straining and unnecessary.

\section{Problem statement}

Available literature on investigations conducted by researchers on the value of emergency nursing students' experiences within the pre-hospital environment as a learning environment is scarce. It was thus essential to explore the value of the pre-hospital environment as a learning environment with the intent to provide evidence for the necessity of pre-hospital placement of emergency nursing students enrolled in the emergency nurse programme. It is vital for emergency nursing students to gain appropriate and related clinical experience, and acquire new knowledge and better rewarded skills. They need to be exposed to and work in various clinical learning environments (of which the prehospital learning environment is just one) because the clinical experience assists them in advancing their current knowledge and skills. When equipped with the applicable, necessary knowledge and skills the emergency nurses become increasingly specialised (Chesser-Smyth, 2005, p. 320; Melby, 2000, p. 638) which will result in better quality care and improved patient outcomes.

This study focused on exploring the value that exposure to the pre-hospital environment added to the personal and professional development of emergency nursing students. In order to maximise the emergency nursing students' clinical learning experience, the researcher believed there was a need to evaluate the experiences of emergency nursing students enrolled in the emergency nursing programme in the prehospital learning environment.

\section{Research method and design}

A qualitative, explorative, descriptive and contextual research design was employed. Data was collected from an Appreciative Inquiry stance. Appreciative Inquiry works on the assumption that there is something good within every programme and that what is good should be carried forward (Hammond, 1998, pp. 20-21). Therefore, the good within the current emergency nursing programme had to be evaluated in support of the pre-hospital rotation. According to Cooperrider, Whitney, and Stavros (2005, p. xviii), Appreciative Inquiry offers an opportunity to build stimulating and dynamic programmes. Appreciative Inquiry is based on a 5D-cycle that provides access to and mobilises the positive core that is encircled by five phases - definition, discovery, dream, design and destiny - with a central positive core (see Fig. 1).

According to Reed (2007, p. 2), Appreciative Inquiry is an inquiry that focuses on appreciating what works well within an organisation (in this case, the emergency nursing programme). Therefore, Appreciative Inquiry is a process that can be utilised to challenge the existing assumptions of an organisation (emergency nursing programme) from a positive stance, by asking questions relating to the strengths and successes of an organisation (emergency nursing programme), or a component of the organisation (programme), with the purpose of developing and implementing an improved plan for the organisation or the 


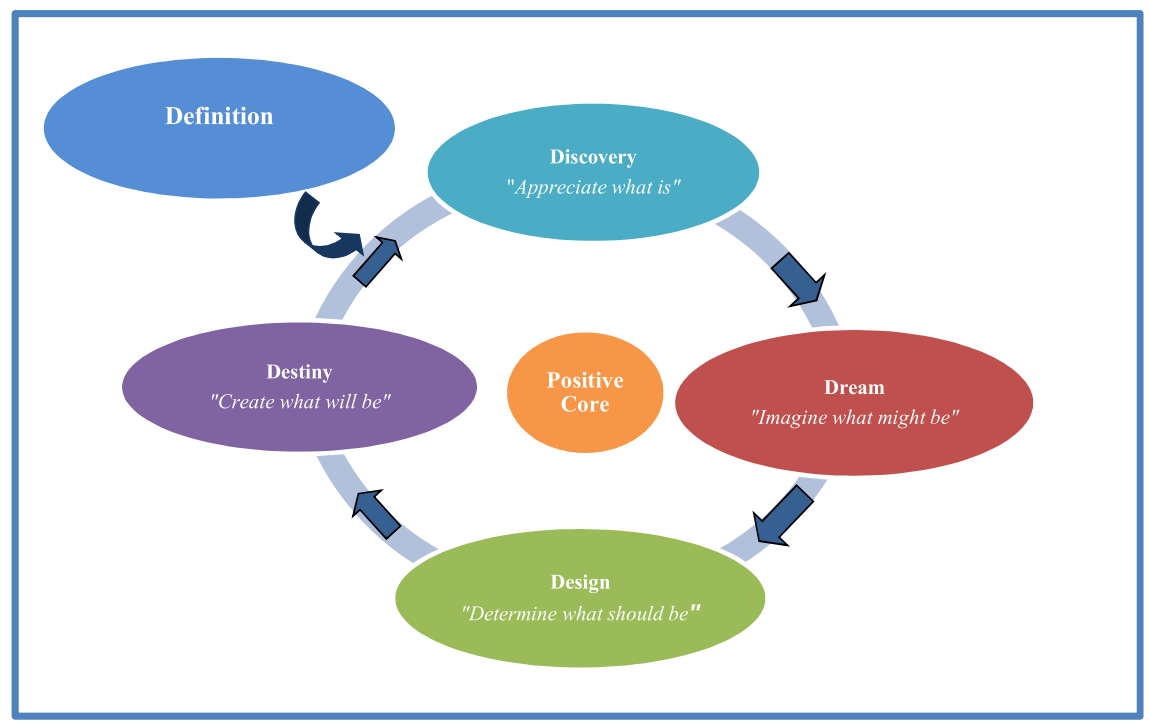

Fig. 1 - Appreciative inquiry - 5D-Cycle. Adapted from Whitney and Trosten-Bloom (2003, p. 6) and Cooperrider et al. (2005, p. 5).

specific component of the organisation (emergency nursing programme).

Before conducting the study, ethical clearance was obtained from the Research Ethics Committee of the Faculty of Health Science of the University of Pretoria and the Department of Nursing Science.

\subsection{Population and sampling}

The population incorporated emergency nurses and emergency nursing students who were enrolled in the emergency nursing programme at a specific nursing education institution. All participants met the following required population criteria:

- They were emergency nurse practitioners who had successfully completed the post-basic qualification in emergency nursing during the past three years.

- They were current emergency nursing students who rotated through the pre-hospital environment.

Participants were identified through purposive sampling. From a total of 60 potential participants who were approached to obtain informed consent, 45 voluntarily participated.

\subsection{Data collection}

Following ethical clearance to conduct the study, the researcher gained access to the population by requesting a list from the nursing education institution of the emergency nursing students who had successfully completed the emergency nursing programme during the previous three years as well as the currently enrolled emergency nursing students. Data was collected using one-on-one Appreciative Inquiry interviews (15 participants) and self-reported Appreciative Inquiry interview schedules (30 participants). An Appreciative Inquiry interview schedule, similar to a questionnaire, was compiled by the researcher with the support from her supervisors to be used as a tool to guide the individual Appreciative Inquiry interviews. An interview is described by Denzin and Lincoln (2005, p. 643) as a conversation where an interviewer poses questions and listens to the participants.

The rationale for including both an Appreciative Inquiry interview and the self-reported Appreciative Inquiry interview schedules was based on the vast geographical distribution of the participants who were eager to participate but unable to travel the long distances for the one-on-one interviews. Using the two data collection techniques contributed to data triangulation and data saturation.

Participants were exposed only to three of the four phases relating to Appreciative Inquiry as the researcher defined the research topic and used the destiny phase for the recommendations to be made based on the participants' contributions (see Table 2).

An information leaflet, informed consent form and Appreciative Inquiry interview schedule were distributed by the researcher in person to participants who would be interviewed and via registered post to those who lived far from the interview setting. A stamped envelope provided by the researcher was included in the mail. The return address was that of a designated secretary at the nursing education institution. Enough time was allowed for all participants to complete, sign and return the forms and the interview schedule. The researcher collected all completed interview schedules from the designated secretary after one month.

\subsection{Data analysis}

The data was classified into categories and subcategories by means of inductive reasoning, phrases and words from the verbatim transcribed interviews and the interview schedules. The data analysis principles as set out by Tesch (1990, pp. 143-145) were followed as qualitative data was collected. Initially the researcher gained a sense of the whole by reading 
through all the collected data. Topics were selected and clusters were made in order to compare topics. The data was reviewed and clusters refined. The data analysis was carried out by the researcher and verified by an independent cocoder.

\section{Ethical considerations}

Ethical clearance was granted by the University of Pretoria Research Ethics Committee as well as the Department of Nursing (S35/2010). The ethical principles considered included beneficence, justice, and respect for human rights which incorporates the right to self-termination, privacy, autonomy and self-confidentiality, fair treatment, and the right to protect from discomfort and harm (Burns \& Grove, 2005, p. 181).

A participant information leaflet was given to each potential participant in which the nature and purpose of the study were thoroughly explained. Participation was entirely voluntary and participants could choose to withdraw from the study at any time. The potential participants were assured that withdrawal from participation would not influence any future studies at the nursing education institution. Every potential participant received a copy of the participation leaflet and the informed consent form. They were also assured of anonymity and confidentiality. All participants provided written informed consent.

\section{Trustworthiness}

Trustworthiness refers to methodological soundness and adequacies of a study and the degree of confidence qualitative researchers have in their criteria (Holloway \& Wheeler, 2002, p. 254; Polit \& Beck, 2008, p. 768). The criteria for trustworthiness as identified by Lincoln and Guba (cited in Polit \& Beck, 2008, p. 539) include (i) credibility, (ii) dependability, (iii) confirmability, (iv) transferability, and (v) authenticity. The strategies used to establish the trustworthiness of this study are summarised in Table 1.

\section{Findings and discussion}

Four main themes emerged from the data. They concerned issues relating to (1) the unpredictable environment, (2) the role players in emergency medical services, (3) team work, and (4) competencies.

\subsection{The unpredictable environment}

The unpredictable pre-hospital environment proved to be valuable to the emergency nursing students. They learnt that it is easy to judge the management provided by paramedics when the latter enter the hospital with a patient, and also that nurses seldom acknowledge the paramedics for their endurance in this unpredictable environment. The emergency nursing students gained insight into the various locations and situations where patients may be in need of emergency care. Some of the unpredictable locations included working on railway tracks and on the open road. The emergency nursing students were exposed to unknown and difficult situations where they were faced with medical and trauma-related emergencies. Working at the home of a patient or with a patient on the road seemed to enhance their understanding and appreciation of the various environmental factors that influence the paramedics' working domain. These environmental factors include working in the rain, in extreme temperatures, and in conditions where the lighting is poor. The following quotes support this finding:

Table 1 - Strategies used to enhance trustworthiness in this study.

\begin{tabular}{|c|c|c|}
\hline Strategy & Criteria & Realisation in the study \\
\hline Credibility & Prolonged engagement & $\begin{array}{l}\text { - The researcher was involved in the emergency nursing practice as well as } \\
\text { a clinical facilitator within the clinical learning environment for } \\
\text { approximately } 10 \text { years. } \\
\text { - Positive open-ended questions were asked in both the self-reported } \\
\text { Appreciative Inquiry interview schedule as well as the Appreciative Inquiry } \\
\text { one-to-one interviews (no full stop). } \\
\text { - Interviews lasted approximately } 30 \mathrm{~min} \text {. }\end{array}$ \\
\hline & Triangulation & $\begin{array}{l}\text { - Utilisation of more than one data collection technique: } \\
\text { O Appreciative Inquiry one-to-one interviews } \\
\text { O Self-reported Appreciative Inquiry interview schedules } \\
\text { O All data was collected by the researcher to enhance consistency }\end{array}$ \\
\hline & Referential adequacy & $\begin{array}{l}\text { - Audio-recording of individual Appreciative Inquiry interviews } \\
\text { - Verbatim transcription of the Appreciative Inquiry interview data }\end{array}$ \\
\hline & Dense description & - Comprehensive description provided of the data collection techniques \\
\hline Transferability & $\begin{array}{l}\text { Purposive sampling } \\
\text { Dependability audit }\end{array}$ & $\begin{array}{l}\text { - Intentional selection of study participants } \\
\text { - Independent coder used }\end{array}$ \\
\hline Dependability & $\begin{array}{l}\text { Dense description } \\
\text { Peer examination } \\
\text { Code-recode procedure }\end{array}$ & $\begin{array}{l}\text { - Comprehensive description of the research methodology followed } \\
\text { - Review by expert supervisors } \\
\text { - Consensus between the researcher, independent coder, and supervisors }\end{array}$ \\
\hline Confirmability & Bracketing & $\begin{array}{l}\text { - Researcher's own beliefs set aside } \\
\text { - Researcher remained open to revealed data }\end{array}$ \\
\hline
\end{tabular}


Table 2 - Questions utilised in the Appreciative Inquiry Interview schedule.

5D-Cycle phase

Discovery

Dream

Design
Question

Reflecting back on the six weeks clinical placement in the pre-hospital environment, what was your most satisfying/peak experience? Imagine it is five years from now and it is now your duty to help improve the pre-hospital learning environment. What would you want to implement?

What is your vision for the six weeks clinical placement in the pre-hospital environment?
- "... disorganised and unusual geographical locations ..."

- "... we had to climb up and down the platform and run on the tracks [railway tracks] ..."

- "... resuscitation on the road is so much different to resus [resuscitation] in the hospital ..."

- "... being exposed to all types of emergencies ..."

- "... exposed to scenes on the road as well as scenes in the home ..."

- "... working in the rain made it difficult to even perform everyday skills such as putting up an IV [intravenous line] ...”

- “... working in temperature extremes as well as inadequate light ..."

Emergency nursing students realised that the pre-hospital environment is a difficult and unpredictable environment (Melby, 2000, p. 642; 2001, p. 773). The remoteness or nearness of the location, a poor infrastructure, and the geographical scenery which can vary between being mountainous to deep valleys and ravines can hinder emergency services (Goosen, 2002, p. 705). The author adds the great distances that sometimes have to be travelled to reach emergency sites in urban areas are barriers in the way of providing emergency services. Schull, Morrison, Vermeulen and Redelmeier (2003, p. 709) expand on this aspect by stating that at times ambulances need to travel through dense traffic and extreme weather conditions in order to reach an emergency site.

Brysiewicz and Bruce (2008, pp. 128-129) observe that emergency nurses in South Africa are exposed to a diversity of significant events accompanied by life-threatening trauma. They come across a wide assortment of compound and difficult clinical conditions in the various clinical environments (Boyle, Williams, Cooper, Adams, \& Alford, 2008, p. 1; Bryant et al., 2003, [1]; Melby, 2001, p. 733). The dissimilarities of the situations and environments where the ambulance services render a service can often be hazardous and unknown; therefore, emergency nursing students need experience in different and opposing environments (Lloyd, 2008, p. 409). When an emergency situation arises in mountainous areas with accompanying climate changes, it can be extremely challenging to reach the site in order to render emergency services and to evacuate the patients (Lischke, Byhahn, Westphal, \& Kessler, 2001, p. 177). Also, factors such as poor lighting in the pre-hospital environment can affect the pre-hospital personnel's effective assessment and management of a patient (Eckstein, Chan, Schneir, \& Palmer, 2000, p. 643).

\subsection{Role players in emergency medical services}

The emergency nursing students learnt more about the various role players within the emergency medical services, specifically the police and fire departments. It led to an increased awareness that the police ensure scene safety, the safety of the personnel, and the patient's safety. The fire department not only assists with the extrication of patients entrapped in vehicles but also with scene safety, the safety of personnel as well as the patient. Quotes that support this theme are the following:

- "... learning from experienced CCAs [critical care assistants] ..."

- "... having the most qualified paramedics on the scene ..."

- "... learning about scene safety from fire officers ..."

- "... fire department also on scene, ensuring the environment is free of hazards ..."

- "... while the door is being cut open by the fire department ..."

- "... [the] traffic was converted by metro police ..."

- "... the police barricaded the road to ensure ..."

Primary assessments and rapid, safe transportation to the nearest appropriate facility of patients are some of the pivotal pre-hospital actions paramedics have to perform on the ill and/or injured (Eckstein et al., 2000, p. 643; Kotze, 1990, p. 320). As a routine, the fire department attends to every traumarelated call where they are in charge of coordinating scene safety (Crawford, Mackway-Jones, Russell, \& Carley, 2004, p. 26; Mackenzie \& Sutcliffe, 2000, p. 40; Malcolm Smith \& Conn, 2009, p. 523). Similar to the fire department, the police play a crucial role in ensuring scene safety. They control the traffic, clear the way for emergency vehicles arriving and leaving the scene, and perform a scene investigation (Band et al., 2010, p. 35).

\subsection{Team work}

Team work was regarded as an important learning curve for the emergency nursing students. They realised the value of working together as a team to ensure the best possible outcome for the patient. It became evident to them that functional and precise communication plays a vital role in ensuring effective team work and a safe outcome for the patient. It includes effective communication from the moment of dispatch to arriving on the scene. All communication from the call centres must be regarded as critical because it leads to rapid responses to the various call-outs. If there is not effective communication from the call centres, the paramedics will not know about any emergencies nor will they know where the emergency is. In addition, communication with the receiving hospital is vital to secure adequate preparation for and receiving of the patient. An important aspect of good communication that the emergency nursing students learnt 
was that mutual respect between the various role players has a positive effect on care delivery and the patient then receives the best possible care:

- “... increased cooperation that existed between emergency unit and EMS [emergency medical service] ..."

- "... team work was excellent ..."

- "... everyone on scene work together ... know what is expected of them ..."

- “... responding to [the] accident scene, in [within] minutes of [a] call ... the police and paramedics worked together to ensure a safe scene..."

- “... contact between pre-hospital team and hospital ensured that EU [emergency unit] personnel were ready for the pt [patient] on arrival ..."

- “... precise communication between dispatch and team and from team to hospital ..."

- "... a better communication structure between the doctors and nurses working in hospital and EMS [emergency medical services] to prevent disagreement ..."

- "... paramedics from different companies, work together ..."

- "... working together [nurses and paramedics]create mutual respect for each other ..."

Team work is critical within the pre-hospital environment; inefficient team work may lead to uncoordinated, fragmented and ineffective care (Melby, 2001, p. 733). Effective multidisciplinary team work is regarded as crucial to ensure safe patient care (Clark, Fisher, Arafeh, \& Druzin, 2010, p. 268) but for this to transpire and also to ensure correct decisions regarding patient care, good communication with and among the multidisciplinary team is pivotal (Castrèn et al., 2008, p. 194; Wong \& Blandford, 2002; [2]). Coordinated continuity of care is ensured by good team work which is not an uncommon phenomenon within the healthcare profession (Hallikainen, Väisänen, Rosenberg, Silfvast, \& Niemi-Murola, 2007, p. 327; Merriman, 2008, p. 57). Furthermore, communicating information received from the call centre to the relevant ambulance personnel is regarded as crucial (Campo, McNutty, Sabatini, \& Fitzpatrick, 2008, p. 153; Gunnarsson \& Stomberg, 2009, p. 86). According to Castrèn et al. (2008, p. 196) and Lockey (2000, p. 7), effective communication by the dispatch centre may reduce the response time of emergency medical services. Clear communication with patients as well as attentively listening to them is considered extremely important (Day, Field, Campbell, \& Reuter, 1995, p. 360) as it can promote quick and precise decision-making in order to administer appropriate and safe care. Working relationships between the paramedics and other healthcare professionals has become more important over time (Kotze, 1990, p. 321); therefore, mutual respect requires mature practitioners as well as a willingness from the team to work closely together in order to overcome any rescue obstacles and to enhance team work (Stohler, 1998, p. 118).

\subsection{Competencies}

Lastly, the emergency nursing students valued the enhanced learning opportunities to practise specific clinical skills with experienced paramedics in order to develop these competencies. The emergency nursing students found that working in the pre-hospital environment benefitted theor$\mathrm{y}$-practice correlation because they acquired more knowledge and developed a deeper understanding of the mechanism of injury as well as the paramedic drug protocols. The emergency nursing students visually observed the various mechanisms of injuries and believed that this knowledge would also be useful to them in hospital. Since the emergency nursing students had to study the paramedic drug protocols before they were exposed to the pre-hospital environment, their understanding of their own practice improved. The emergency nursing students found that they had also learned more with regard to the triage of patients; they felt this would help them become independent practitioners. They were able to improve their assessment skills, had a better understanding of continuous management and haemodynamic monitoring of the patient from the moment of first contact with the stabilisation of patients to preparation for transport as well as the delivery of the patients to the hospital. Supporting quotes include:

- "... proper handling of an injured patient and secure protection after extracted ... as well as the monitoring of patient on the way to hospital ..."

- "... the triage of the patient on scene helped us to determine which patient to treat first ..."

- "... I was able to develop assessment skills - was able to think for myself ..."

- “... we [emergency nurses] now have an appreciation for mechanism of injury ... understand why it is important to learn about it ..."

- "... understanding of how to manage the patient ..."

- "... to maintain C-spine [cervical spine] ... doing it on the scene made us [emergency nurses] understand why we practise it in the skills lab [laboratory] and that one should maintain the C-spine [cervical spine] ... starting where the patient sits in the care ..."

- "... asked me to assess the patients ... although it was in the patient's home ... I [emergency nurse] realised that I could do it ..."

- "... I developed an appreciation for the knowledge and skills of the paramedics ..."

- "... learnt the importance of mechanism of injury ..."

- "... [I] develop assessment skills ... I was left on my own to assess and manage a patient ... this gave me so much confidence ..."

- "... the triage of the pt [patient] on scene was regarded as very important ... if we [emergency nursing students and paramedics] did not triage patients, we [emergency nurses and paramedics] would not know who to treat first ..."

- "... correct and effective patient assessment and management was vital ... we [emergency nurses and paramedics] do not have any backup ... I [emergency nurses] realised this was one skill that I [emergency nurse] needed to practise to perfection ..."

- "... the monitoring of patient on the way to hospital in the back of an ambulance made me realise how important it was to know the equipment as well as how difficult it was when the vehicle [ambulance] was moving ..."

- "... the paramedic protocols helped ... we [emergency nurses] felt safe as we knew what to do and in which order to do it ..." 
To ensure the correlation of theory with practice, clinical placement is regarded as an integral part of nursing education (Baglin \& Rugg, 2010, p. 145; Jerlock et al., 2003, p. 221; Sendir \& Acaroglu, 2008, p. 737). Students are thus able to identify their own learning needs within the clinical practice as they apply the various theories taught in the classroom (Crooks et al., 2005 , p. 364). According to Koh (2002, p. 40), clinical placement can be viewed as a way of enhancing the theor$\mathrm{y}$-practice correlation. Protocols can be seen as a guide utilising physiology, anatomy and mechanism of injury to guide decisions made by paramedics (Smith \& Conn, 2009, p. 25). Clinical placement allows students the opportunity to master their procedures (Baglin \& Rugg, 2010, p. 147) and allows for the application and interpretation of protocols (Edwards et al., 2004, p. 249). Practice guidelines for the paramedics in South Africa are set out in their protocols by the Health Professional Council of South Africa.

In terms of Maslow's hierarchy of needs, students have a need to know and understand (Mellish, Brink, \& Paton, 2005, p. 35). The student's need for knowing and understanding is satisfied once they know and understand, and the more they know and understand, the more they want to know and understand. The clinical placement of nursing students also helps them to develop an increased understanding of their own career (Arbon, 2004, p. 152; Baglin \& Rugg, 2010, p. 147). Equally important is that clinical placement allows emergency nursing students a better understanding of the emergency medical services as well as the care they provide (Melby, 2000, p. 642; 2001, p. 731).

\section{Limitations}

The study mainly fulfilled its aim, namely to explore the value that exposure to the pre-hospital environment as part of the emergency nursing programme has for emergency nursing students. As with any research studies, some limitations need to be noted.

The study was limited to only one tertiary nursing education institution. Many of the students who had successfully completed the emergency nurse programme from 2008 to 2011 did not reside in the same city as where the tertiary nursing education institution was located. Not only did this situation make it difficult to obtain perspectives from a multitude of informants, but it necessitated the researcher to make use of additional data collection strategies which is not optimal when using an Appreciative Inquiry approach. Despite the fact that the Appreciative Inquiry interview schedule utilised the principles that guide Appreciative Inquiry, no dialogue between the researcher and the participants was possible. Finally, the study was limited to only one specific tertiary nursing institution in Gauteng, South Africa; the findings are thus limited to only one geographical area.

\section{Recommendations}

From the study results it appears that rotating through the pre-hospital learning environment provides valuable learning opportunities and augments team work. It is therefore recommended that emergency nursing students enrolled for an emergency nursing programme should rotate through the pre-hospital environment in spite of possible cost implications for private hospital companies. It is also recommended that similar research projects be undertaken at other tertiary nursing education institutions in order to determine the true value of pre-hospital rotation throughout the emergency nursing programmes offered in South Africa. It may be interesting to compare the value of pre-hospital rotation in South Africa with that of other countries. In addition, it may be useful to evaluate the team dynamics between emergency nurses and various other role players in the emergency environment more comprehensively.

\section{Conclusion}

This research provided insightful feedback from the participants with regard to their rotation through the pre-hospital learning environment. Clinical exposure was found to be a valuable and significant learning experience for the emergency nursing students. They were able to make use of their competencies and they came to value the disciplined and good team work they encountered. It is therefore clear that rotating through the pre-hospital learning environment provided the emergency nursing students with fundamentally valuable learning opportunities.

\section{Competing interests}

The authors declare that they have no financial or personal relationship(s) which may have inappropriately influenced them in writing this article.

\section{Authors' contributions}

S.v.W. (University of Pretoria) conducted the research as part of a Master's degree in Emergency Nursing Science and wrote the manuscript. T.H. (University of Pretoria) and I.C. (University of Pretoria) were the study leaders and provided feedback on and approval of the article.

\section{Acknowledgements}

This research was undertaken for the requirements of an MCur (Clinical) in Emergency Nursing Science.

\section{R E F E R E N C E S}

Arbon, P. (2004). Understanding experience in nursing. Journal of Clinical Nursing, 13(2), 150-157.

Baglin, M. R., \& Rugg, S. (2010). Student nurses' experiences of community-based practice placement learning: a qualitative exploration. Nurse Education in Practice, 10(3), 145-152.

Band, R. A., Pryor, J. P., Gaieski, D. F., Dickinson, E. T., Cummings, D., \& Carr, B. G. (2010). Injury-adjusted mortality 
of patients transported by police following penetrating trauma. Academic Emergency Medicine, 21(1), 32-37.

Boyle, M. J., Williams, B., Cooper, J., Adams, B., \& Alford, K. (2008). Ambulance clinical placements - a pilot study of students' experience. Medical Education, Open Access, 8, 1-6. Retrieved from http://0-www.biomedcentral.com.innopac.up.ac.za/ content/pdf/1472-6920-8-19.pdf.

Bryant, P., Hartley, S., Copolla, W., Berlin, A., Modell, M., \& Murray, E. (2003). Clinical exposure during clinical method attachments in general practice. Medical Education, 37(9), 790-793.

Brysiewicz, P., \& Bruce, J. (2008). Emergency nursing in South Africa. International Emergency Nursing, 16(2), 127-131.

Burns, N., \& Grove, S. K. (2005). The practice of nursing research: Conduct, critique and utilization (5th ed.). St. Louis, Missouri: Elsevier Saunders.

Campo, T., McNutty, R., Sabatini, M., \& Fitzpatrick, J. (2008). Nurse practitioners performing procedures with confidence and independence in the emergency care setting. Advanced Emergency Nursing Journal, 30(2), 153-170.

Castrèn, M., Karlsten, R., Lippert, F., Christensen, E. F., Bovim, E., Kvam, A. M., et al. (2008). Recommended guidelines for reporting on emergency medical dispatch when conducting research in emergency medicine: the utstein style. Resuscitation, 79(2), 193-197.

Chesser-Smyth, P. A. (2005). The lived experiences of general student nurses on their first clinical placement: a phenomenological study. Nurse Education in Practice, 5(6), 320-327.

Chung-Heung, L., \& French, P. (1997). 'Education in the practicum: a study of the ward learning climate in Hong Kong. Journal of Advanced Nursing, 26(3), 455-462.

Clark, E. A. S., Fisher, J., Arafeh, J., \& Druzin, M. (2010). Team training/simulation. Clinical Obstetrics and Gynecology, 53(1), 265-277.

Cooperrider, D. L., Whitney, D., \& Stavros, J. M. (2005). Appreciative inquiry handbook: The first in a series of AI workbooks for leaders of change. San Fransisco: Berrett-Koehler Publishers.

Crawford, I. W. F., Mackway-Jones, K., Russell, D. R., \& Carley, S. D. (2004). Delphi based consensus study into planning for chemical incidents. Emergency Medical Journal, 21(1), 24-28.

Crooks, D., Carpio, B., Brown, B., Black, M., O'Mara, L., \& Noesgaard, C. (2005). Development of professional confidence by post diploma baccalaureate nursing students. Nurse Education in Practice, 5(6), 360-367.

Day, R. A., Field, P. A., Campbell, I. E., \& Reuter, L. (1995). Students evolving beliefs about nursing: from entry to graduation in a four-year baccalaureate programme. Nurse Education Today, 15(5), 357-364.

Denzin, N. K., \& Lincoln, Y. S. (Eds.). (2005). The sage handbook of qualitative research (3rd ed.). Thousand Oaks, California: Sage.

Eckstein, M., Chan, L., Schneir, A., \& Palmer, R. (2000). Effects of pre-hospital advanced life support on outcomes of major trauma patients. The Journal of Trauma: Injury, Infection and Critical Care, 48(4), 643-648.

Edwards, H., Smith, S., Courtney, M., Finlayson, K., \& Chapman, H. (2004). The impact of clinical placement location on nursing students' competence and preparedness for practice. Nurse Education Today, 24(4), 248-255.

Goosen, J. (2002). The malignant epidemic - changing patterns of trauma. South African Medical Journal, 92(10), 798-802.

Gunnarsson, B. M., \& Stomberg, M. W. (2009). Factors influencing decision making among ambulance nurses in emergency care situations. International Emergency Nursing, 17(2), 83-89.

Hallikainen, J., Väisänen, O., Rosenberg, P. H., Silfvast, T., \& Niemi-Murola, L. (2007). Interprofessional education of medical students and paramedics in emergency medicine. ACTA Anaesthesiologica Scandinavica, 51(3), 372-377.

Hammond, S. A. (1998). The thin book on appreciative inquiry (2nd ed.). Plano, Texas: Thin Book Publishing.

Holloway, I., \& Wheeler, S. (2002). Qualitative research in nursing (2nd ed.). London: Blackwell.

Jackson, D., \& Mannix, J. (2001). Clinical nurses as teachers: Insights from students of nursing in their first semester of study. Journal of Clinical Nursing, 10(2), 270-277.

Jerlock, M., Falk, K., \& Severinsson, E. (2003). Academic nursing education guidelines: tools for bridging the gap between theory, research and practice. Nursing and health sciences, 5(3), 219-228.

Koh, L. C. (2002). The perceptions of nursing students of practicebased teaching. Nurse Education in Practice, 2(1), 35-43.

Kotze, J. M. (1990). The role of the ambulance service as part of the health profession. South African Medical Journal, 78, 320-321.

Lischke, V., Byhahn, C., Westphal, K., \& Kessler, P. (2001). Mountaineering accidents in the European Alps: have the numbers increased in recent years? Wilderness and Environmental Medicine, 12(2), 74-80.

Lloyd, A. (2008). Informing practice: information experiences of ambulance officers in training and on-road practice. Journal of Documentation, 66(3), 396-419.

Lockey, D. J. (2000). Pre-hospital trauma management. Resuscitation, 49, 5-15.

Mackenzie, M. R., \& Sutcliffe, M. R. (2000). Pre-hospital care: the trapped patient. Journal of Royal Army Medical Corps, 2000(146), 39-46.

Malcolm Smith, R., \& Conn, A. K. T. (2009). Pre-hospital care scoop and run or stay and play. Injury: International Journal of the Care of the Injured, 40(4), 23-26.

Melby, V. (2000). Experiential learning in pre-hospital emergency care: a qualitative study. Nurse Education Today, 20(8), 638-645.

Melby, V. (2001). The adrenaline rush: nursing students' experiences with the Northern Ireland ambulance service. Journal of Advanced Nursing, 34(6), 727-736.

Mellish, J. M., Brink, H. I. L., \& Paton, F. (2005). Teaching and learning the practice of nursing (4th ed.). Johannesburg: Heinemann.

Merriman, M. L. (2008). Pre-hospital discharge planning: empowering elderly patients through choice. Critical Care Nurse, 31(1), 52-58.

Meyer, S., \& van Niekerk, S. (2008). Nurse educator in practice. Cape Town: Juta.

Polit, D. F., \& Beck, C. T. (2008). Nursing research: Generating and assessing evidence for nursing practice (2nd ed.). Philadelphia: Lippincott Williams \& Wilkins.

Reed, J. (2007). Appreciative inquiry. Research for change. India: Sage.

Sendir, M., \& Acaroglu, R. (2008). Reliability and validity of Turkish version of clinical stress questionnaire. Nurse Education Today, 28(6), 737-743.

Schull, M. J., Morrison, L. J., Vermeulen, M., \& Redelmeier, D. A. (2003). 'Emergency department gridlock and out-of-hospital delays for cardiac patients'. Academic Emergency Medicine, 10(7), 709-716.

Smith, R. M., \& Conn, A. K. T. (2009). Pre-hospital care - scoop and run or stay and play? Injury: International Journal of the Care of the Injured, 40(4), 23-26.

South African Nursing Council. (1993). Regulations relating to the course in clinical nursing science leading to registration of an additional qualification, R212 as amended. Retrieved from http:// www.sanc.co.za/regulations/Reg-cln.htm.

Stedman, T. L. (2012). Stedman's medical dictionary for health professions and nursing. Philadelphia: Wolters Kluwer Health/ Lippincott Williams \& Wilkins.

Stohler, S. A. (1998). High performance team interaction in an air medical program. Air Medical Journal, 17(3), 116-120. 
Tesch, R. (1990). Qualitative research: Analysis types and software tools. Basingstoke, Hampshire: The Falmer Press.

Whitney, D., \& Trosten-Bloom, A. (2003). The power of appreciative inquiry: A practical guide to positive change. San Francisco: Berret-Koehler Publisher Inc.
Wong, B. L. W., \& Blandford, A. (2002). Analysing ambulance dispatcher decision making: Trailing emergent themes analysis. Retrieved from http://0-eprints.ucl.ac.uk.innopac.up.ac.za/ $16628 /$. 